\title{
Highly Efficient Generation of Strong-Field 0.1-THz Radiation
}

\author{
Xiaojun $\mathrm{Wu}^{1,2}$, Anne-Laure Calendron ${ }^{1,2}$, Koustuban Ravi ${ }^{1,4}$, Chun Zhou ${ }^{1,3}$, Michael Hemmer ${ }^{1}$, Fabian \\ Reichert $^{1,3}$, Dongfang Zhang ${ }^{1,3}$, Huseyin Cankaya ${ }^{1,2}$, Luis E. Zapata ${ }^{1}$, Matlis, Nicholas ${ }^{1}$, and Franz X. \\ Kärtner ${ }^{1,2,3,4}$ \\ ${ }^{1}$ Center for Free-Electron Laser Science, DESY, 22607 Hamburg, Germany \\ ${ }^{2}$ Center for Ultrafast Imaging, University of Hamburg, 22607 Hamburg, Germany \\ ${ }^{3}$ Department of Physics, University of Hamburg, 22761 Hamburg, Germany \\ ${ }^{4}$ Department of Electrical Engineering and Computer Science and Research Laboratory of Electronics, MIT, \\ Cambridge, Massachusetts 02139, USA
}

\begin{abstract}
We demonstrate the generation of single-cycle 0.1$\mathrm{THz}$ radiation in a lithium niobate crystal using optical rectification and obtain $\mathrm{THz}$ output energy of $65 \mu \mathrm{J}$ with 31.6 MV/m peak field. This amounts to an optical-to-THz energy conversion efficiency of $0.3 \%$ at $85 \mathrm{~K}$, which is in agreement with theory.
\end{abstract}

$\mathrm{T}$ he sub-terahertz $(\mathrm{THz})$ frequency range $(v=0.1-1 \mathrm{THz}$, $\lambda=0.3-3 \mathrm{~mm}$ ) is becoming increasingly essential for exploring basic science, such as plasma diagnostics, molecular spectroscopy, and also for industrial applications, such as non-invasive inspection and environmental sensing due to its higher transmittance in soft materials and in the atmosphere compared to $\mathrm{THz}$ waves $(>1.0 \mathrm{THz})$ and higher directivity than microwaves. This low frequency range is also interesting for electron acceleration and therefore compact coherent x-ray sources [2]: the short wavelengths and the high gradients are promising to provide exceptional characteristics of the electron bunches, e.g. in terms of emittance.. Lower frequencies can accommodate higher electron bunch charges than higher ones that were used for demonstrating electron acceleration [2]. In addition, $0.1 \mathrm{THz}$ waves may also be useful for electron bunch diagnostics using deflecting cavities and therefore can provide a new indirect $\mathrm{x}$-ray detection method. In order to accelerate electrons to relativistic energies from rest, $\mathrm{THz}$ pulses at the mJ-level are required. Using optical schemes like difference frequency generation, which have efficiencies in the 0.1 to $1 \%$ range, Joule-level laser systems are then required. Cryogenically cooled Yb:YAG laser amplifiers are a promising technology for this application as they can produce high-energy picosecond pulses with $0.5 \mathrm{~nm}$ bandwidths at $\mathrm{kHz}$ repetition rates, which are ideal for generating $\mathrm{THz}$ pulses in the $0.1 \mathrm{THz}$ range.

We have already generated high energy, strong-field singlecycle $\mathrm{THz}$ pulses with high optical-to-THz energy conversion efficiencies of percent- and half-percent levels from $1030 \mathrm{~nm}$ and $800 \mathrm{~nm}$ wavelengths, respectively, using broadband phase matching via tilted pulse fronts (TPF) in lithium niobate crystals [1]. For the first time to our knowledge, we apply this method to generate intense $0.1 \mathrm{THz}$ pulses, which corresponds to the bandwidth of the above mentioned $\mathrm{Yb}$ :YAG long laser pulses.

THz pulses were generated by sending up to $21-\mathrm{mJ}$ of $4.7-$ ps, 1030-nm Yb:YAG laser pulses into a lithium niobate (LN) crystal in a tilted pulse front phase-matching geometry, as shown in Fig. 1(a). The characteristics of the IR driver (autocorrelation, spectrum and beam profile) are shown in Fig. 1(b) and (c). Following a grating with 1500 lines $/ \mathrm{mm}$ placed at $56.2^{\circ}$ angle of incidence, an imaging system onto the output surface of the LN prism demagnify the beam by a factor of -0.5. To decrease the linear absorption, the LN crystal was cryogenically cooled down to $85 \mathrm{~K}$. The $\mathrm{THz}$ beam was collected for energy measurements and for imaging of the near-field onto a pyroelectric camera. The output $\mathrm{THz}$ energy is measured by a pyroelectric $\mathrm{THz}$ detector (Gentec SDX-1152) and a ZnTe crystal is used to perform electrooptical sampling. The performed simulations of the $\mathrm{THz}$ generation, based on a $(2+1 \mathrm{D})$ code and accounting for all spatio-temporal distortions of the IR beam, confirmed the experimental results.

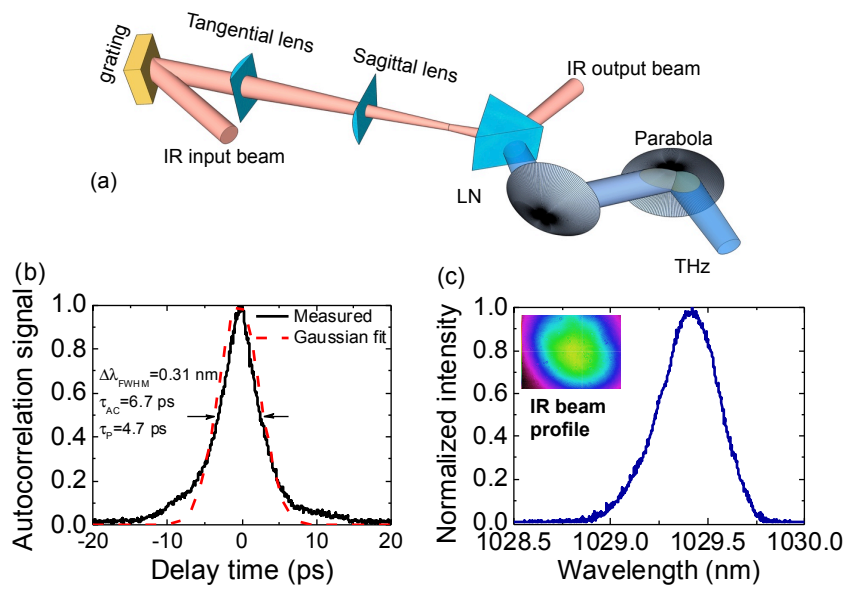

Fig.1. (a) Layout of the experimental setup. LN: lithium niobate prism. The $\mathrm{THz}$ beam is imaged with 2 OAPs. The laser delivers $4.7 \mathrm{ps}$ pulses, as shown in the autocorrelation (b) corresponding to nearly transform limited pulses the spectrum is shown in (c) with the beam profile in inset.

As shown in Fig. 2(a), an optical-to-THz energy conversion efficiency of $0.3 \%$ is achieved at cryogenic temperature, resulting in THz pulses of $65 \mu \mathrm{J}$. This efficiency is close to theoretical predictions of $0.34 \%$. At room temperature, $0 . .08 \%$ conversion efficiency was achieved. Due to the cascading process, the IR output is spectrally broadened when the IR photons down convert to $\mathrm{THz}$ photons, as seen in Fig. 2(b)measured at room temperature. The simulation of the broadened spectrum is in qualitative agreement with our measurement. 

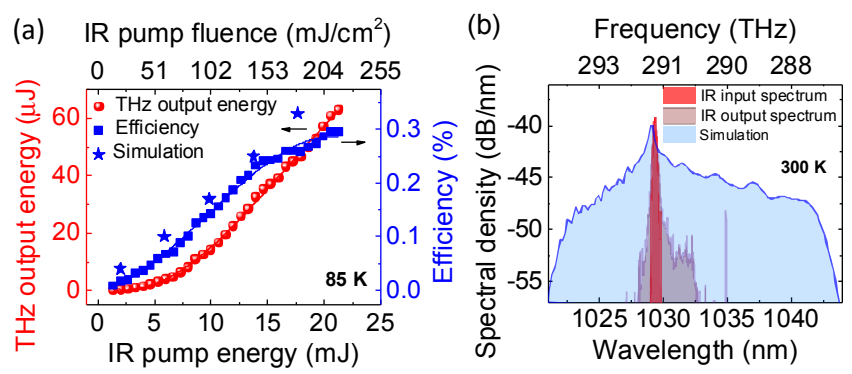

Fig.2. (a) $\mathrm{THz}$ output energy and corresponding conversion efficiency as a function of pump energy and fluence, measured at cryogenic temperature. (b) Measured IR input and output spectra and expected output IR spectrum from simulations after $\mathrm{THz}$ generation.

Figure 3(a) shows the THz temporal waveform measured via electro-optic sampling and its corresponding spectrum. The generated single-cycle $\mathrm{THz}$ pulses have a long pulse duration of $\sim 10 \mathrm{ps}$ and the peak frequency is located at $0.1 \mathrm{THz}$, close to the predicted frequency. The spectral shape is in general agreement with the theory.

For strong-field experiment, a high beam quality is suitable. The image of the beam onto a camera, presented in the inset of Fig. 3(b), shows a nearly circular beam profile. The cascading process can affect the beam profile: nonlinear distortions depend on the fluence of the driving beam. The measurement of the THz spatial profile in the far-field shows in this setup no strong distorsion. The measurement of the spatial profile at several places along the propagation caustique allowed us to estimate the divergence of the beam to $\sim 21^{\circ}$. This is close to diffraction limit for a waist of $\sim 2.5 \mathrm{~mm}$. We also focused the beam to $2.5 \mathrm{~mm}$, the smallest demagnification achievable with the available optics.

Based on the measured $\mathrm{THz}$ energy, pulse duration and beam size $\left(2.5 \mathrm{~mm} 1 / \mathrm{e}^{2}\right.$ diameter $)$, the estimated $\mathrm{THz}$ peak field reaches $31.6 \mathrm{MV} / \mathrm{m}$. The $\mathrm{THz}$ beam is intense and we can visualize it by eye on a liquid crystal plate.

Besides our interest in electron acceleration and strong-field $\mathrm{THz}$ generation experiments, this single-cycle, strong-field $0.1 \mathrm{THz}$ source, presenting an excellent beam quality, is also important for nonlinear $\mathrm{THz}$ field-matter interaction and spectroscopy. We expect this source can open up new ways for cutting edge research and broader applications in the $\mathrm{THz}$ region.
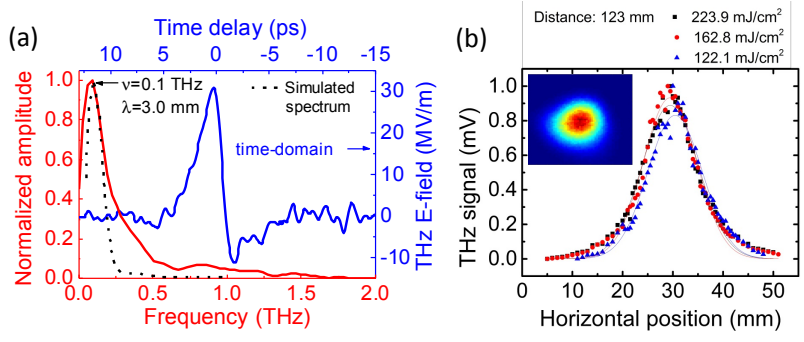

Fig.3. (a) The measured THz temporal waveform and its spectrum by electrooptic sampling. (c) Measurement of the beam profile along the horizontal direction for different driving IR intensities, prone to nonlinear distortions. The inset shows the focused $\mathrm{THz}$ beam.

\section{References}

[1]. J. Hebling, G. Almási, I. Z. Kozma, and J. Kuhl, "Velocity matching by pulse front tilting for large-area THz pulse generation," Opt. Expresss 101161 (2002)

[2] E. A. Nanni, W. R. Huang, K.-H. Hong, K. Ravi, A. Fallahi, G. Moriena, R. J. D. Miller, and F. X. Kärtner, Nat. Commun. 6, 8486 (2015).

[3] F. Blanchard, X. Ropagnol, H. Hafez, H. Razavipour, M. Bolduc, R. Morandotti, T. Ozaki, and D. G. Cooke, Opt. Lett. 39, 4333 (2014). 